\title{
Vitamin D deficiency in children: A review on prevention and treatment strategy
}

\author{
*Ibrahim Dughaim Alreshidi \\ Department of Pharmacy, Maternity and Children Hospital, Hafar Al-Batin, Saudi Arabia
}

\begin{abstract}
As an essential fat-soluble vitamin in human body, the main function of vitamin D (VD) is mediated by its receptor. In the past decade, VD has drawn gradual and consistent attention from the scientific community and health professionals. According to epidemiological survey, approximately one billion people are under the state of vitamin D insufficiency or deficiency in the world. This phenomenon is also very common in China. By participating in calcium and phosphorus absorption from GIT, VD stands as a very important functional immune regulator. It plays a vital role in modulating both innate and adaptive immune responses, mainly through the modulation of growth, differentiation and function of a variety of immune cells, and the influence of secretion of cytokines and antimicrobial peptides. VD deficiency and pediatric diseases such as acute lower respiratory infection and asthma are associated with not only pediatric skeletal disorders (including nutritional rickets, osteomalacla, and osteoporosis), but also pediatric non-skeletal disorders, such as mortality rate, cardiovascular disorders and mortality, metabolic syndrome (including obesity, impaired glucose tolerance (IGT), diabetes mellitus, lipid metabolism disorders and hypertension), cancer, infection, allergy and asthma, neurological and mental diseases, autoimmune diseases, and chronic kidney diseases. Although VD treatment of skeletal disorders has been widely recognized, its efficacy and long-term effect on non-skeletal disorders is not clear. Hence, for the benefit of general children and their families, aim of the present review is to determine some convincing facts of evidence-based medicine about Chinese children, and to discuss certain reasonable, right and effective measures to prevent VD deficiency thereby reducing the risk of rickets.
\end{abstract}

Key Words: Vitamin D, children, Chinese, non-skeletal disorders, prevention, pediatric.

\section{INTRODUCTION}

It is generally believed that the problem of vitamin $\mathrm{D}(\mathrm{VD})$ deficiency has been elucidated, but recent survey showed that 50 percent of the global population is still under risk of vitamin D deficiency (Euphytica, 2014). The key reason of causing VD deficiency is people mistakenly believe that by sunlight exposure or dietary supplements, they can gain enough VD, but ignore the importance of VD supplementations. Children are potential high risk of VD deficiency group that can make them susceptible to rickets, causing growth retardation and skeletal deformities and may increase osteoporosis in adults and elderly hip fracture risks (Paediatr Perinat Epidemiol, 2014). VD deficiency has also been related to cancer, autoimmune diseases, endocrine system diseases, hypertension and infectious diseases. This review shades light on general knowledge about the VD, its impact on the pediatric diseases, complicacy arises by high dose of VD, way to handle the situation and finally, summarizes how maintaining a balanced VD level can lead to healthy life.

\section{OVERVIEW OF VITAMIN D}

\section{The source of vitamin D}

There are three known sources of vitamin D for infants: (1) the maternal fetal transformation; (2) food intake and (3) light synthesis of the skin. In the strictly sense, VD is not a common type of vitamin, it not only can be taken with diet, but also can be produced by the skin when ultraviolet rays from sunlight strikes it. Sufficient dosage of $\mathrm{VD}$ is very difficult to achieve from diet only. It is

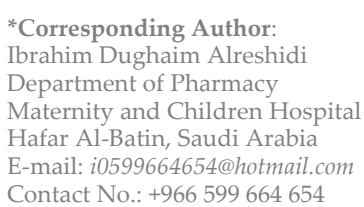

clearly unrealistic and difficult for the newborns, infants and young children to get enough VD by ultraviolet irradiation to fulfill their growth and development requirement. In north China, the temperature is low in winter, ultraviolet ray is weak, and the daily sunshine time is short. Moreover, atmospheric pollution in the area limits skin exposure to the sunlight and affects skin synthesis of vitamin D. Therefore, it is essential to add extra VD for the infants who are under the age of 3 , especially less than 1 year old. VD, sourced from skin synthesis or food intake, is mainly stored in adipose tissue.

\section{The mechanism of vitamin $D$}

VD does not directly effect on target organs to play a physiological role, however it goes into the blood after absorption and upon binding to specific globulin (DBP), it flushes into liver, through the 25-hydroxylase it becomes 25-hydroxyl vitamin D (25 OHD), and then 1,25- $(\mathrm{OH})_{2} \mathrm{D}$, which is formed through the 1-a hydroxylase of kidney. This has strong biological activity, and its mechanisms of action are similar to that of steroid hormone; biological effects are mediated by specific cell receptor (VDR) (Pediatr Allergy Immunol, 2014).

\section{The metabolism of vitamin $D$}

Vitamin D is a fat-soluble sterol derivative; there are mainly two forms of it: vitamin D2 and vitamin D3. When vitamin $\mathrm{D}$ flushes into liver, through the 25 - hydroxylase, it becomes 25 - hydroxyl vitamin $\mathrm{D}, 25-(\mathrm{OH}) 2 \mathrm{D}$ generated for vitamin $\mathrm{D}$, which is the main forms of storage and circulation in our body. In the kidney, under the effect of 1 alpha hydroxylase, it generates $1,25-(\mathrm{OH}) 2 \mathrm{D}$, in which $1,25-(\mathrm{OH}) 2 \mathrm{D} 3$ is the main form of activity (American Journal of Ophthalmology, 2014). 
The evaluation index of vitamin $D$

Now it is believed that the level standard of children serum $25(\mathrm{OH}) \mathrm{D}$ is as follows: $75 \mathrm{nmol} / \mathrm{L}$ is the ideal level, $50-75 \mathrm{nmol} / \mathrm{L}$ shows vitamin $\mathrm{D}$ insufficiency, $<75 \mathrm{nmol} / \mathrm{L}$ shows vitamin $\mathrm{D}$ deficiency, $12.5 \mathrm{nmol} / \mathrm{L}$ shows a severe lack of vitamin $\mathrm{D}$. On the other hand, $>250 \mathrm{nmol} / \mathrm{L}$ shows exceeding amount of vitamin $\mathrm{D}$ and $>375 \mathrm{nmol} / \mathrm{L}$ shows vitamin D intoxication.

\section{THE RELATIONSHIP BETWEEN VITAMIN D AND PEDIATRIC CLINICAL DISEASE}

\section{Rickets}

The lack of VD and calcium intake can cause convulsions in children, but VD deficiency rickets and childhood calcium nutritional deficiencies are two different concepts. Although the former is involved in the problem of calcium and phosphorus metabolism, it is not equivalent to calcium deficiency rickets. VD deficiency rickets is caused by the lack of VD which cause abnormalities of calcium and phosphorus metabolism, leading to incomplete mineralization of bone tissue when children are growing, then produce chronic systemic nutritional diseases characterized by bone lesions and closely related to our life. Rickets disease not only affects children's growth and development, but also associated with VD deficiency. The disease is one of the four key pediatrics preventable diseases in our country (The Journal of Nutrition, 2014). Children grow faster in infantile period, infants is fastest growing stage in life. There are reasons that cause nutritional VD deficiency rickets, inadequate intake, short sun exposure time, premature births and so on. They also make the infant become high-risk groups of vitamin D insufficiency. This should be dealt with high importance by children's doctors, pediatricians and parents.

\section{Respiratory diseases}

Vitamin D is a kind of new immunomodulatory hormone, mainly in immunoregulation of our body. When body is deficient of VD, it causes obstacle of calcic phosphor metabolization and decrease of phosphoruscalciumphosphorus. Many biochemical reactions are ceased or reduced, cellular and humoral immune function decreased, and it suppresses autoimmune reaction, leading to various types of infections, especially recurrent respiratory tract infections. Because of children's soft ribs and muscles, respiratory functions are affected. It raises the risk of incidence of lung infection, called "rickets lung".

\section{Immuno regulation and immune disease}

In addition to regulating calcium and phosphorus metabolism, 1, 25- $(\mathrm{OH})_{2} \mathrm{D}$ also have extensive effects on the immune systems. Most of cells related to immune system contain VD receptors (VDR); it is various to regulate the immune systems, including regulating the proliferation of lymphocyte and the secretion of cytokines, etc.; it also plays an important role in the occurrence and development of infectious diseases and immune diseases (Annals of Human Biology, 2014). 1,25-(OH) $2 \mathrm{D}$ would also encourage monocytes to differentiate into macrophages, which is the first barrier of the nonspecific immunity. In clinical practice, malnourished children are more likely to develop respiratory and alimentary, and this also shows that nonspecific immunological function is at a low level.

\section{Obesity}

Studies show that the levels of 25-OHD are reduced in overweight or obese individuals and abdominal obesity. There was an independent inverse correlation between serum 25-OHD and the body mass index and waist circumference. It suggests obesity is closely related to the fall of the VD level. It is clear that levels of serum 25-OHD were lower from the obese people than the non-obese people. While under the same light condition, obese individuals' bioavailability of synthetic VD of skin falls $50 \%$ from non-obese individuals as its adipose tissue is more, and VD is fat-soluble. It is reserved by adipose tissue for use. There is less VD released into the circulation of blood, resulting in lower levels of serum VD.

\section{Infection}

Inherent immune cells can be induced to express antimicrobial peptides by VD. Antimicrobial peptides possess antibacterial and antiviral activity. VD deficiency can lead to the decrease of body's defense reaction, which is one of the reasons why may be prone to infection. Studies have shown that low serum VD levels can obviously increase the risk of active pulmonary tuberculosis (OR, 0.68; 95\% CI, 0.43 0. 93). And studies show that about $97 \%$ of TB patients' serum $25(\mathrm{OH}) \mathrm{D}$ is below $30 \mathrm{ng} / \mathrm{ml}$.

\section{Renal diseases}

VD is used in the clinical treatment of chronic renal insufficiency, including in the application of patients with chronic kidney disease (CKD) stage 3 4 and the dialysis patients (Pediatr Dermatol, 2015). Vitamin D can use inhibit rennin-angiotensin - aldosterone system and inflammatory factor and so on to reduce proteinuria and glomerular sclerosis, in addition, a quarter of children's have idiopathic simple hematuria disease because of idiopathic hypercalciuria. What's more, some patients, because of 1- $\alpha$-hydroxylase dysfunction, cause serum $1,25-(\mathrm{OH})_{2} \mathrm{D}$ levels and urinary calcium excretion to rise.

\section{APPLICATION DEFECT OF VD IN PEDIATRIC CLINIC}

Excessive administration of VD results in its accumulation and may cause hypercalcaemia and nephrocalcinosis. It can cause other electrolyte disorder, retarded growth and development, which influence intelligence function of children and affect their heart, lung, kidney and gastrointestinal functions, even lead to dispersion metastatic renal and vascular calcification.

\section{Hypercalcinemia}

Vitamin D plays an important role in the process of calcium absorption. Vitamin D is steroidal derivatives, and it plays the role of resistance of rickets, which also call for the vitamin of resistance of rickets. However, in recent years due to improper supplement the calcium in infants, there happened many hypercalcemia caused by VD intoxication, which VD may be effective inducer of building hypercalcemia model. VD adjust a variety of physiological functions through promote intestinal absorption of calcium and phosphorus (European Journal of Nutrition, 2013). The initial symptoms of hypercalcemia is weakness, tiredness, low-spirited, headache, nausea, vomiting, diarrhea, etc., and then renal function disorders and appear polydipsia, diuresis, proteinuria, urine concentrated force decreases, likewise, there will be multiple tissues calcium calm and influence organizational function. For example, renal calcium composure will 
hurt renal, which first renal tubular functions disorders and then influence the glomeruli figure. If those symptoms are severe, it will lead to heart artery calcification and cause death (Nutrition Bulletin, 2013).

\section{Renal calcium ectopic composure}

Some studies have shown that large dose of VD lead to hypercalcemia, and metastatic calcium deposits in the lungs, at the bottom of the gastric mucosa gland, glomerular and convoluted tubule and so on, which those organs usually discharge acidic material, but its own organization is basophilic. Therefore, intracellular calcium caused by high-dose vitamin D 3 deposits have cytotoxic effect that cause those tissue cell degeneration, necrosis, loss of epithelial cells blocking malpighian tube cavity, which very easily come into being renal calculus and calcification was found in the kidney tissues (Journal of the Academy of Nutrition and Dietetics, 2013), and so high doses of vitamin D preparation can cause infant viscera ectopic calcification and continuous kidney damage. Renal damage appear frequent urination, urine specific gravity and low fixed, proteinuria, urinary leukocytosis and can cause renal failure, and arterial hypertension.

\section{Cardiovascular calcification}

Excessive intake of vitamin D make visible pericardial have milky calcified plaque in the heart, and there are multiple focal necrosis and calcium deposits in the myocardium. The damaged cardiac could appear auscultation hearing noise. Through ECG check, S T segment elevation can be seen. Severe case may cause congestive heart failure and some children have been diagnosed with weight loss, debilitating fatigue and are prone to infection and fever. Coronary artery and the necrosis of its branches of middle cell and vascular intima and endocardium have calcium deposits as well as heart valve and chordae tendineae have calcification. Additionally, the aorta is irregular expansion, and the artery wall is uneven, which have a milky calcium spot. Middle artery smooth muscle dies and has a lot of crumb calcium deposits.

\section{Corneal calcification}

With systemic VD poisoning, conjunctiva and cornea demonstrate typical changes with epithelium and under epithelium calcium salt deposition as well as edema, necrosis and desquamation. Maculae cornea infer calcium salt, and the cornea malnutrition change, what is more, the structure lesions is possibility as same as too much calcium salt. Ball conjunctival vessel in the palpebral fissure appear hyperemia, when through the slit lamp examination, we can discover palpebral fissure place close to the limbal conjunctival surface have mild edema (European Journal of Nutrition, 2013), which show hyaline changes, like transparent crystal. Besides, the lesion area can be seen white line deposition, which will cover some the blood vessels, some patients bleed around the corneal limbus. The change of cornea is thin white epithelium and epithelial cell line nubecula, and the nubecula appear the nasal side and temporal side, which perpendicular to the corneal limbus, and the corneal limbus slightly broaden and uneven here.

\section{Other influence}

Using too much vitamin D may cause some other GIT complications, for example, anorexia (especially for solid diet), nausea and vomiting, dehydration, acidosis, constipation, acute ulcer pain, acute gland inflammation etc. Spiritual consciousness includes irritation, crying and spirit of euphoria. Elderly children are encountered with headache, followed by lassitude, depression or lethargy. For a long time chronic poisoning can cause viscera or soft tissue calcification and the corresponding symptoms is corneal calcification. Besides, it may also cause renal calcification, calcification around joints, blood vessels calcification and calcified cartilage calcification.

Thus, when VD is overused resulting decreased appetite, vomiting, constipation, weakness and slow growth, blood calcium concentration should be checked. If the concentration is obviously higher than normal, VD supplementation should be immediately stopped until the symptoms disappeared. The therapy can be continued afterward with correct dosage regime.

\section{CONCLUSION}

With the increasing knowledge about the importance of proper VD level in the body and risk associated with its excessive level, scientific research institutions, academic groups and teaching hospital should carry out profound and wide studies with reasonable study design. To benefit the general children and their families, reasonable, right and effective preventive measures should be taken for preventing vitamin D deficiency and to reduce the risk of vitamin D rickets. In daily life, to satisfy requirements of vitamin D in children, they should be provided with plenty of sunshine, reasonable dietary supplement and if situation demands, vitamin D enhancer can be applied. In the early childhood (Journal of the Academy of Nutrition and Dietetics, 2013), active prevention and treatment of vitamin $\mathrm{D}$ deficiency can prevent the occurrence of rickets, and reduce the bone fracture risk of adulthood. Moreover, ensuring adequate vitamin D nutrition in the childhood can effectively reduce some adulthood diseases that are difficult to cure such as malignant tumor, type-1 diabetes, the incidence of hypertension. But at the same time, careful attention should be given not to overdose the vitamin D supplements, in order to avoid the phenomenon of vitamin D intoxication.

\section{REFERENCES}

Au, L.E., Harris, S.S., Jacques, P.F., Dwyer, J.T., Sacheck, J.M., (2014).

Adherence to a vitamin d supplement intervention in urban schoolchildren. J Acad Nutr Diet, 114(1), 86-90. [DOI]

Cara L. Eckhardt,Alison D. Gernand,Daniel E. Roth,Lisa M. Bodnar. Maternal vitamin D status and infant anthropometry in a US multicentre cohort study. Annals of Human Biology,2014,24(23):290-291.

Ekaterina Maslova, Susanne Hansen, Andrew L. Thorne - Lyman, Camilla B. Jensen, Marin Strøm, Arieh Cohen, Nina O. Nielsen, Sjurdur F. Olsen. Predicted vitamin D status in mid-pregnancy and child allergic disease. Pediatr Allergy Immunol,2014,25(17):1093-1094. [DOI]

Enoch G. Achigan-Dako, Olga E. D. Sogbohossou, Patrick Maundu. Current knowledge on Amaranthus spp.: research avenues for improved nutritional value and yield in leafy amaranths in sub-Saharan Africa. Euphytica,2014,19(07):1209-1210. [DOI]

Lauren E. Au, Susan S. Harris, Paul F. Jacques, Johanna T. Dwyer, Jennifer M. Sacheck. Adherence to a vitamin D supplement intervention in urban schoolchildren. Journal of the Academy of Nutrition and Dietetics, 2013,09(14):932-933.

Lin Chen, Xinke Chen, Qin Xiang, Yuqiang Zheng, Lianhong Pi, Qin Liu, Jun Xiao, Ning Ke, Jing Fang. Prevalence of Low Serum Vitamin A Levels in Young Children with Chalazia in Southwest China. American Journal of Ophthalmology,2014,21(09):980-981. [DOI]

Nicola Principi,Sonia Bianchini,Elena Baggi,Susanna Esposito. Implications of maternal vitamin D deficiency for the fetus, the neonate and the young infant. European Journal of Nutrition,2013,05(23):1922-1923. [DOI]

Pinkaew, Siwaporn, Wegmuller, Rita, Wasantwisut, Emorn, Winichagoon, Pattanee, Hurrell, Richard F, Tanumihardjo, Sherry A. Triple-Fortified Rice Containing Vitamin A Reduced Marginal Vitamin A Deficiency 
and Increased Vitamin A Liver Stores in School-Aged Thai Children1-3. The Journal of Nutrition,2014,14(04):484-485.

Prentice, A. Standing on the shoulders of giants: Understanding calcium and vitamin D requirements. Nutrition Bulletin,2013,13(08):1123-1124. [DOI]

Principi Nicola, Bianchini Sonia, Baggi Elena, Esposito Susanna. Implications of maternal vitamin D deficiency for the fetus, the neonate and the young infant. European Journal of Nutrition,2013,21(15):854855.

Sarah A. Keim,Lisa M. Bodnar,Mark A. Klebanoff. Maternal and Cord Blood 25(OH)-Vitamin D Concentrations in Relation to Child Development and Behaviour. Paediatr Perinat Epidemiol,2014,28(05):783-784. [DOI]
Shekhar Neema, Sweta Mukherjee, Biju Vasudevan, Rajesh Verma, Nikhil Moorchung, Manas Chatterjee. Vitamin D Deficiency After Oral Retinoid Therapy for Ichthyosis. Pediatr Dermatol, 2015,32(14):275-276. [DOI]

Thornton Kathryn A,Marín Constanza,Mora-Plazas Mercedes,Villamor Eduardo. Vitamin D deficiency Associated with Increased Incidence of Gastrointestinal and Ear Infections in School-Age Children.. The Pediatric Infectious Disease Journal,2013,09(12):693-694. [DOI] 